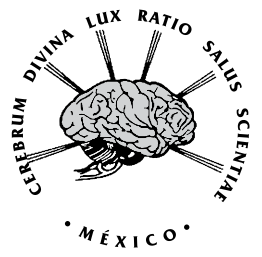

\title{
Farmacoepidemiología de los fármacos antagonistas de los receptores de angiotensina (ARA) II en el tratamiento de la hipertensión arterial esencial. México
}

\section{Pharmacoepidemiology of antagonist receptors of the angiotensin (ARA) II in the treatment of essential hypertension. Mexico}

\author{
Adela Alba-Leonel, ${ }^{*}$ Guillermo Fajardo-Ortiz, ${ }^{\star *}$ Joaquín Papaqui-Hernández ${ }^{\star \star *}$
}

* Maestra en Ciencias Sociomédicas con énfasis en Epidemiología, Profesora de Carrera Asociado "C", Escuela Nacional de Enfermería y Obstetricia, Universidad Nacional Autónoma de México.

** Médico Cirujano, Master of Hospital Administration, Maestría en Ciencias Administrativas y Doctor en Historia, Profesor Titular "C", Facultad de Medicina, Universidad Nacional Autónoma de México.

*** Ingeniero en Informática, y Especialista en Estadística Aplicada, Instituto Mexicano de Seguridad Social.

\section{Palabras clave: \\ Hipertensión \\ arterial esencial, \\ ARA II, eficacia de \\ Ios ARA II, IECA, \\ farmacoepide- \\ miología.}

\section{RESUMEN}

Epidemiología de la hipertensión arterial: A nivel mundial, se reporta que la prevalencia de hipertensión arterial en población adulta se encuentra entre un 20-27\%. Tratamiento farmacológico: Los ARA II ejercen su acción a través del bloqueo de los receptores AT1 de la angiotensina II de forma específica y altamente selectiva y actúan inhibiendo los efectos clásicos desfavorables de angiotensina II. Así, los ARA II antagonizan la acción de la angiotensina II producida por cualquier vía enzimática y no actúan sobre la degradación de las quininas, por lo que no condicionan un aumento de sus niveles en sangre. Además, al bloquear los receptores ATI los ARA II permiten que la angiotensina II circulante se pueda ligar a los receptores AT2, lo que puede añadir un efecto adicional potencialmente muy beneficioso. Estudios de morbimortalidad en Hipertensión con ARA II: La eficacia de los ARA II para disminuir la tensión arterial está demostrada desde hace varios años, tanto en HTA leve como moderada o severa, y en múltiples estudios comparativos estos fármacos muestran una eficacia antihipertensiva similar a la de los IECAS, antagonistas del calcio o betabloqueantes. Por tanto, ante una eficacia antihipertensiva similar y un mejor perfil de tolerabilidad, los ARA II pueden considerarse una familia farmacológica de gran utilidad en el tratamiento actual de la HTA. Conclusiones: La conclusión de los autores de diversos estudios es que los IECA y los ARA-II tienen una efectividad antihipertensiva similar y los IECA, una peor tolerabilidad.

\section{ABSTRACT}

Key words:

Essential

hypertension, ARA II, efficacy of $A R A$ II, IECA inhibitors, pharmacoepidemiology.
Epidemiology of hypertension: Epidemiology of hypertension globally, it is reported that the prevalence of hypertension in adult population is between 20-27\%. Pharmacotherapy: ARA II exert their action by blocking AT1 receptors Angiotensin II in a specific and highly selective and act by inhibiting the adverse effects angiotesin II classic. Thus, antagonize the action of angiotensin II produced by any means and do not act on enzymatic degradation of kinins, so do not condition an increase in blood levels. In addition to the ATI receptor blockade ARA II allow the circulating Angiotensin II may be linked to the AT2 receptor, which can add an additional potentially beneficial. Studies of morbidity and mortality in hypertension with ARA II: The efficacy of ARA II for lowering blood pressure is well established for several years, both in mild hypertension and moderate or severe and multiple studies comparing these drugs have shown similar antihypertensive efficacy to that of IECA inhibitors, calcium antagonists or beta-blockers. Therefore, given similar antihypertensive efficacy and better tolerability profile the ARA II may be considered a very useful drug family in the current treatment of hypertension. Conclusions: The conclusion of the authors of several studies is that IECA inhibitors and ARA II have similar antihypertensive effectiveness and IECA inhibitors, poorer tolerability.

Recibido para publicación: 25/04/2014. Aceptado: 01/08/2014.

Este artículo puede ser consultado en versión completa en: www.medigraphic.com/enfermerianeurologica 


\section{EPIDEMIOLOGÍA DE LA HIPERTENSIÓN ARTERIAL}

A nivel mundial, la OMS reporta que la hipertensión arterial (HTA) causa la muerte de 7.5 millones de personas, lo que representa el $12.8 \%$ del total de las muertes; ${ }^{1}$ así mismo señala que en uno de cada tres adultos que tienen la presión alta ${ }^{2}$ se generan complicaciones como insuficiencia cardiaca, enfermedad vascular periférica, insuficiencia renal, retinopatía y discapacidad visual.

La prevalencia de HTA, a nivel mundial, es de $27 \%$ en población adulta. Por regiones, África tiene la prevalencia más alta, con $36.8 \%$, y la región de las Américas la más baja, con $23 \%$. En la región de Canadá y Estados Unidos se tienen prevalencias bajas, con alrededor de $15 \%$, seguidas por México (24.4\%), y en Brasil, Chile y Cuba $30 \%$, aproximadamente, respecto a la población de 25 años y más. ${ }^{2-4}$

Actualmente, México está en un periodo de transición epidemiológica avanzada, enfrentando simultáneamente el desafío de la carga de las enfermedades crónicas y los rezagos de patologías infecciosas..$^{5-7}$ El perfil demográfico se caracteriza por una urbanización e industrialización aceleradas; en las últimas décadas se han incrementado los procesos migratorios en los cuales gran parte de la población rural se ha trasladado a núcleos urbanos, lo que ha repercutido en su condición de vida, hábitos y estilos de vida. ${ }^{8}$

A principios de los 90 , el perfil epidemiológico del país muestra predominio de las enfermedades no transmisibles e incremento en la proporción de muertes atribuibles a enfermedades crónicas como principal causa de defunción. ${ }^{9}$ Este fenómeno está asociado al envejecimiento de la población y al desarrollo de riesgos relacionados con estilos de vida poco saludables. Las enfermedades crónicas han tenido un impacto mayor en la mortalidad por enfermedades del corazón, HTA, tumores malignos y diabetes mellitus, entre otras. ${ }^{5}$

La HTA es un padecimiento cada vez más frecuente que se asocia con otras enfermedades e incluso es también causa de muerte, por lo que se considera un problema de salud pública. La Encuesta Nacional de Salud 2000 (ENSA) ${ }^{10}$ reportó una prevalencia a nivel nacional de $30.7 \%$. En el 2006, la Encuesta Nacional de Salud y Nutrición (ENSANU) ${ }^{11}$ reportó un incremento de 12.5 puntos porcentuales, teniendo una prevalencia de HTA de $43.2 \%$ en población de 20 años y más, y la ENSANU del $2012^{12}$ reportó una prevalencia de $31.5 \%$, ocupando la séptima causa de morbilidad para el mismo año. ${ }^{13}$ Cabe señalar que del total de hipertensos sólo el $36.6 \%$ recibe tratamiento y de éstos sólo el $25 \%$ están controlados.

Por otro lado, se ha demostrado que en mayores de 45 años de edad el $90 \%$ de los casos desarrolla HTA esencial y menos del $10 \%$ HTA secundaria; si analizamos los cambios en la composición de la estructura demográfica de la población observamos que tanto la prevalencia como la incidencia van a tener una tendencia ascendente en las próximas décadas. ${ }^{9,14}$
Al analizar el peso que tuvo la HTA sobre la mortalidad en México durante 2005, encontramos que hubo 493,957 decesos ocurridos: 93,434 (18.9\%) fueron defunciones asociadas a la presencia de HTA.

La farmacoepidemiología comenzó a desarrollarse en los EUA en la década de los 60 con los programas de monitorización de reacciones adversas a los medicamentos en los hospitales como el John Hopkins Hospital y The Boston Collaborative Drug Surveillance Program, que consistieron en estudios de cohortes donde se exploraron los efectos a corto plazo de medicamentos en los hospitales.

\section{TRATAMIENTO FARMACOLÓGICO}

Los antagonistas de los receptores de la angiotensina II (ARA II) ejercen su acción a través del bloqueo de los receptores AT1 de la angiotensina II de forma específica y altamente selectiva y actúan inhibiendo los efectos clásicos desfavorables de angiotensina II. Así, los ARA II antagonizan la acción de la angiotensina II producida por cualquier vía enzimática y no actúan sobre la degradación de las quininas, por lo que no condicionan un aumento de sus niveles en sangre. Al bloquear los receptores ATI, los ARA II permiten que la angiotensina II circulante se pueda ligar a los receptores AT2, lo que puede generar un efecto adicional potencialmente beneficioso. ${ }^{15}$ Por otra parte, la bradiquinina circulante, además de sus efectos vasodilatadores, parece estar asociada a ciertos efectos adversos como la tos seca, proceso que limita el uso de inhibidores de la enzima convertidora de angiotensina (IECA) hasta en un $5-20 \% .{ }^{16}$ Los ARA II, por tanto, están libres de esta reacción adversa.

Algunos estudios demuestran que los ARA II tienen una tolerabilidad mejor que la de otros fármacos antihipertensivos. De hecho, Bloom publicó en 1998 un análisis retrospectivo de 21,700 pacientes tratados con distintos fármacos antihipertensivos y analizó la persistencia del tratamiento con la droga inicial a 12 meses de seguimiento. Este autor observó que los pacientes tratados con un ARA II al año tenían una persistencia en el tratamiento superior respecto a los pacientes tratados con otros fármacos. ${ }^{17}$ Posteriormente, Conlin publicó un seguimiento de 15,000 pacientes del estudio de Bloom desde los 12 a los 48 meses, donde encontró que la continuidad del tratamiento inicial seguía siendo mejor con ARA II. ${ }^{18}$ Se ha demostrado que los ARA II presentan menos efectos adversos que los IECA, principalmente debido a la ausencia de tos, y la incidencia de reacciones adversas de estos fármacos es comparable al placebo. ${ }^{19-21}$ Este excelente perfil de tolerabilidad puede ser fundamental para lograr una mayor adherencia al tratamiento. ${ }^{22}$

La eficacia de los ARA II para disminuir la presión arterial (PA) está demostrada desde hace varios años, tanto en HTA leve como moderada o severa; ${ }^{23-26}$ en múltiples estudios comparativos estos fármacos han demostrado una eficacia antihipertensiva similar a la de los IECA, antagonistas del calcio o betabloqueantes. ${ }^{27-32}$ Por tanto, ante 
una eficacia antihipertensiva similar y un mejor perfil de tolerabilidad, los ARA II pueden considerarse una familia farmacológica de gran utilidad en el tratamiento actual de la HTA. ${ }^{33}$ Las recomendaciones de la OMS-SIH de 1999 establecen que los ARA II deben considerarse, al igual que los betabloqueantes y diuréticos, antagonistas del calcio, IECA o alfabloqueantes como tratamiento de primera elección en HTA. ${ }^{34}$

Por la eficacia, tolerabilidad y los beneficios evidenciados en los pacientes con nefropatía diabética (estudios IDNT y RENAAL) y en los pacientes con hipertrofia ventricular izquierda (estudio LIFE) e HTA, la indicación de estos fármacos se puede ampliar en un futuro inmediato. La American Diabetes Association ha decidido considerar a los ARA II como tratamiento de primera elección en los pacientes hipertensos con diabetes tipo 2 , con microalbuminuria o proteinuria. ${ }^{35}$

Hasta la fecha son siete los ARA II aprobados para HTA: losartán, valsartán, candesartán, irbesartán, eprosartán, telmisartán y olmesartán. Estos fármacos tienen un mecanismo de acción idéntico y sólo difieren en cuanto a sus características farmacológicas. Se han realizado diversos estudios comparativos analizando el efecto sobre la PA; los resultados obtenidos han demostrado algunas diferencias entre ellos en cuanto a la potencia antihipertensiva. ${ }^{36-39}$ Sin embargo, un metaanálisis que agrupó a 11,281 pacientes de 43 estudios diferentes con ARA II no mostró diferencias significativas en cuanto a la reducción de PA alcanzada con los distintos ARA II, por lo que sugiere que los ARA II podrían tener una eficacia similar cuando se administran las dosis usuales recomendadas. ${ }^{37}$

\section{ESTUDIOS DE MORBIMORTALIDAD EN HIPERTENSIÓN CON ARA II}

La incidencia de complicaciones cardiovasculares relacionadas con la HTA sigue siendo alta, a pesar de las numerosas alternativas terapéuticas disponibles en el mercado y a las diversas estrategias de tratamiento instauradas. En los últimos años se han realizado varios estudios sobre morbimortalidad cardiovascular en el paciente hipertenso comparando distintos fármacos entre sí. Principalmente se compararon los efectos de los fármacos "modernos" como antagonistas del calcio e IECA frente a los fármacos "clásicos" como betabloqueantes y diuréticos. Al contrario de lo que se pensaba, no se observaron diferencias significativas entre los fármacos en términos de morbimortalidad cardiovascular. ${ }^{40-43}$ Estos hallazgos parecen indicar que todos los antihipertensivos son similares y el único efecto beneficioso es la reducción de PA. En pacientes hipertensos de riesgo muy alto cardiovascular, como son aquellos con nefropatía diabética, los estudios IDNT y RENAAL realizados con irbesartán y losartán, respectivamente, demostraron una reducción significativa de la morbimortalidad cardiovascular. En ambos estudios, la variable principal de eficacia fue la combinación de muerte, tiempo de duplicación de creatinina sérica y caída en insuficiencia renal terminal. En el estudio IDNT los pacientes se distribuyeron en tres grupos de tratamiento: placebo, amlodipino e irbesartán, donde se demostró que el tratamiento con irbesartán a los 2.6 años de tratamiento produjo una reducción del riesgo relativo del $23 \%(p=0.006)$ respecto a amlodipino y del $20 \%$ ( $p=0.024)$ respecto a placebo, siendo los resultados con amlodipino y placebo similares. ${ }^{44} \mathrm{El}$ estudio RENAAL comparó el tratamiento basado en losartán frente a placebo. También se confirmó, de forma similar al IDNT, una reducción del riego relativo del 16\% $(p=$ 0.024 ) en el grupo tratado con ARA II. ${ }^{45} \mathrm{EI}$ hecho de que en dos ensayos clínicos similares con dos ARA II sean diferentes los resultados, pero tan parejos, confirma los hallazgos y establece la utilidad de estos fármacos en la protección cardio-vasculo-renal del paciente con nefropatía diabética.

Tres grandes estudios de morbimortalidad que analizan el papel de los ARA II en HTA: el estudio LIFE, SCO$P E$ hasta ahora la evidencia disponible es la que proviene del estudio LIFE $^{46}$ que se diseñó hace varios años con la hipótesis de que el antagonismo selectivo de los receptores ATI de la angiotensina II con losartán podría ser más eficaz que el betabloqueo con atenolol para reducir la morbilidad y mortalidad cardiovascular en los pacientes con HTA (PA sistólica 160-190 y/o diastólica 95-110) y signos electrocardiográficos de hipertrofia ventricular izquierda, detectada por criterios del producto duraciónvoltaje de Cornell (>2.440 mm x msg) o por criterios de voltaje de Sokolow-Lyon (> $38 \mathrm{~mm}$ ). (Texto incongruente.) Se realizó una comparación aleatorizada, doble ciego y con doble enmascaramiento de los efectos a largo plazo de losartán con los de atenolol. La variable principal de valoración fue la morbilidad y la mortalidad cardiovasculares, que se definieron por la combinación de muerte de causa cardiovascular, infarto agudo de miocardio e ictus. ${ }^{38}$ Fueron aleatorizados 9,194 pacientes con una edad media de 67 años y una PA media de $174.4 / 98.7 \mathrm{mmHg}$ y se realizó un seguimiento mínimo de 4 años. El basal, demostró que cerca del 16\% había tenido manifestaciones de enfermedad coronaria, $8 \%$ ictus previo o accidente isquémico transitorio y el $13 \%$ eran diabéticos. ${ }^{47,48}$ En estos pacientes los betabloqueantes son una opción adecuada para realizar la comparación con losartán, ya que estos fármacos son de primera elección en el tratamiento de la HTA, tienen una eficacia antihipertensiva similar a losartán ${ }^{49}$ y han demostrado disminuir la morbilidad y mortalidad al compararse con placebo. ${ }^{50} \mathrm{~A}$ los 12 meses de seguimiento, la PA había disminuido a 150.5/85.1 $\mathrm{mmHg}$ pero solamente el $23 \%$ de los pacientes estaba tomando el fármaco de estudio en monoterapia. ${ }^{51}$ Esta tendencia se mantuvo y al finalizar el estudio, sólo el 9-10\% de los pacientes estaban tratados solamente con la medicación inicial (50 mg atenolol o $50 \mathrm{mg}$ de losartán), lo que enfatiza la necesidad de la politerapia en la gran mayoría de los pacientes hipertensos.

A los cinco años se observó que el grupo de pacientes con tratamiento basado en losartán mostró una reducción 
relativa del riesgo del $13 \%$ respecto al grupo de atenolol de aparición de muerte cardiovascular, infarto de miocardio o ictus (508 pacientes del grupo losartán y 588 pacientes del grupo atenolol, $p=0.021)$. Esta reducción del riesgo fue del $25 \%$ en la incidencia de ictus ( $p=$ 0.001 ), ya que la aparición de muerte cardiovascular y de infarto de miocardio fue similar en ambos grupos de tratamiento. La reducción del riesgo era mayor $(14.6 \%, p$ $=0.009$ ) sin introducir un ajuste respecto a la puntuación de riesgo de Framingham y el grado de hipertrofia ventricular izquierda (HVI) por ECG en situación basal. En cuanto a la HTA, hubo una reducción de PA importante y muy similar en ambos grupos $(30.2 / 16.6 \mathrm{mmHg}$ en el grupo losartán y $29.1 / 16.8 \mathrm{mmHg}$ en el grupo atenolol). Esto evidencia que los beneficios adicionales obtenidos por el ARA II son independientes del puro efecto sobre la PA. Secundariamente, el estudio LIFE mostró una reducción significativa del $25 \%(p<0.001)$ del riesgo de padecer diabetes de nueva aparición en los pacientes tratados con losartán frente a los tratados con atenolol. Se observó, además, una mayor regresión de la hipertrofia ventricular izquierda en el grupo de losartán. Con losartán se observó una reducción del 10 y 16\% con los criterios de Comell y de Sokolow-Lyon, mientras que con atenolol la reducción fue de 4.4 y $9 \%$ respectivamente (en ambos casos, $p<0.0001)$. Hay que destacar que esta mayor capacidad de regresión de la hipertrofia ventricular no explica todo el efecto favorable del ARA II respecto a atenolol. Se demostró también una menor incidencia de efectos adversos en el grupo de losartán. ${ }^{44}$ En el subestudio del LIFE que evaluó la morbilidad y mortalidad cardiovascular en 1,195 pacientes diabéticos, los beneficios de losartán fueron aún más pronunciados en todas las variables analizadas, sobre todo en un pequeño grupo de pacientes que no habían sido tratados por HTA. ${ }^{52}$ En este subgrupo de pacientes se observó una reducción del riesgo en la variable primaria del 24\% (103 pacientes asignados a losartán y 139 a atenolol, $p=0.031)$. Además se observó una reducción significativa del $37 \%$ de riesgo de muerte cardiovascular (fallecieron 38 y 61 pacientes de los grupos de losartán y atenolol respectivamente, $p$ $=0.028$ ) y una reducción del $39 \%$ del riesgo de muerte por cualquier causa (64 y 104 muertes, $p=0.002$ ). Estos hallazgos contrastan con los del estudio UKPDS, en el que se comparó el efecto de captopril y de atenolol en pacientes hipertensos diabéticos. En este estudio no se observaron diferencias significativas en morbimortalidad entre el IECA y el betabloqueante. ${ }^{53}$ Por tanto, los beneficios observados en el LIFE con un ARA II, no observados antes con IECA, distinguen el efecto de estas dos clases farmacológicas.

El subestudio del LIFE se realizó en un subgrupo de pacientes con hipertensión sistólica aislada; alrededor del $15 \%$ del total demostró un beneficio mayor en esta población que en los diabéticos. La variable primaria se redujo en $25 \%$, el riesgo de ictus se redujo de forma significativa en un $40 \%$ y el de muerte cardiovascular en un $46 \%$. Además, losartán disminuyó la hipertrofia ventricu- lar izquierda más que atenolol $(p=0.001)$ y fue mejor tolerado. ${ }^{54}$

Un análisis secundario del estudio LIFE examinó clínicamente: la influencia del control de PA sobre la regresión de la hipertrofia ventricular izquierda determinada por ecocardiografía, tras dos años de tratamiento antihipertensivo. Se realizaron controles ecocardiográficos al año y a los dos años y los resultados mostraron que si bien la PA disminuía significativamente durante el primer año, no se modificaba significativamente durante el segundo. Sin embargo, la regresión de la hipertrofia fue persistente y significativa durante los dos años, por lo que disminuyó la prevalencia de hipertrofia concéntrica y excéntrica y aumentó la proporción de pacientes con geometría ventricular normal. Esto demuestra los beneficios adicionales del efecto de reducción de PA y la regresión de la hipertrofia, lo que requiere como mínimo dos años de tratamiento antihipertensivo. ${ }^{55,56}$

Se han publicado diversos subestudios del estudio LIFE que han puesto en evidencia los beneficios del tratamiento antihipertensivo sobre la función sistólica, ${ }^{57}$ la función diastólica, ${ }^{58}$ correlación de la presión de pulso durante el tratamiento antihipertensivo, ${ }^{59}$ correlación del tamaño de la aurícula izquierda, ${ }^{60}$ y la relación del índice albuminuria/creatinina con la función y estructura del ventrículo. ${ }^{61}$

De todos los hallazgos, el resultado más importante del LIFE fue que por primera vez una clase farmacológica, los ARA II (losartán) se ha mostrado superior a otra clase de antihipertensivos (como los betabloqueantes, y en concreto el atenolol). Probablemente el efecto sobre la PA no haya tenido mayor influencia en la incidencia de eventos cardiovasculares, y fundamentalmente de ictus, ya que en ambos grupos la PA fue muy similar. De hecho, la PA sistólica en el grupo de losartán fue $1.3 \mathrm{mmHg}$ más baja que en el grupo de atenolol y la PA diastólica $0.4 \mathrm{mmHg}$ más alta en la última revisión realizada. Estos hallazgos nos llevan a considerar que la presión de pulso final fue algo menor en el grupo de losartán. Este dato podría tener alguna implicación pronóstica en la aparición de ictus, aunque difícilmente puede explicar los beneficios alcanzados con el ARA II, ya que la diferencia de presión entre ambos grupos es muy pequeña. Por otra parte, en el subgrupo de pacientes diabéticos éstos presentaron una mayor presión de pulso que la población en general, y sin embargo, la incidencia de ictus se dio en un pequeño número de pacientes y las diferencias entre los grupos de tratamiento no fueron significativas. Es importante resaltar que en el grupo de pacientes aleatorizados a atenolol había 25 más con fibrilación auricular, lo que podría tener alguna influencia en la aparición de ictus durante el seguimiento.

Los mecanismos por lo que los ARA II han mostrado ser especialmente útiles en los pacientes de mayor riesgo no son bien conocidos. Quizá pueda influir el probable efecto benéfico de estos fármacos en la disfunción endotelial y en los procesos de arteriosclerosis. De todas maneras se ha sugerido que los resultados obtenidos en 
el estudio LIFE podrían quizá ser extrapolados también a pacientes de bajo riesgo. ${ }^{62}$

En un estudio publicado se demuestra que la mayoría de los pacientes hipertensos que acuden actualmente a la consulta de atención primaria en nuestro país son de alto riesgo o muy alto, de acuerdo a la estratificación propuesta por la OMS-SIH de $1999 .{ }^{63}$ Por lo anterior, se ha sugerido que los beneficios observados con losartán podrían ser superiores si se administrara una dosis constante de 100 mg, ya que en el estudio LIFE menos del 50\% alcanzaron esta dosificación y se ha visto que algunos ARA II tienen mayores beneficios a dosis máximas. ${ }^{64}$

El estudio SCOPE es el primer ensayo clínico realizado a gran escala para evaluar los efectos del tratamiento con un ARA II (candesartán) sobre eventos cardiovasculares mayores (infarto de miocardio, ictus y mortalidad cardiovascular) y función cognitiva en pacientes ancianos. ${ }^{65}$ Se trata de un estudio prospectivo, aleatorizado, doble ciego de 4,937 pacientes de ambos sexos de 70 a 89 años de edad (76 años de media), con HTA (PA sistólica 160-179 mmHg y/o diastólica $90 / 99 \mathrm{mmHg}$ ) y de riesgo cardiovascular, principalmente medio y alto. Los pacientes se distribuyeron aleatoriamente a candersartán o placebo. Hay que destacar que debido a la necesidad de tratar la HTA en algunos pacientes y los cambios que han venido sucediendo en cuanto a los objetivos de control de PA, en el grupo placebo casi el $85 \%$ de los pacientes estaba con medicación antihipertensiva, por lo que realmente no puede considerarse que ese grupo de pacientes estuviera en placebo. Tras un seguimiento mínimo de tres años se demostró una reducción no significativa en el riesgo de acontecimientos cardiovasculares mayores en pacientes tratados con ARA II. Sin embargo, se observó una reducción significativa del $28 \%$ del riesgo de ictus ( $p$ $=0.04$ ) en el grupo de pacientes tratados con candersartán. El tercer gran ensayo clínico en HTA con ARA II es el estudio VALUE. ${ }^{66}$ Se trata de un estudio prospectivo, multicéntrico, randomizado, doble ciego, con dos ramas de tratamiento: amlodipino y valsartán. La hipótesis es que a los mismos niveles de control de PA, el ARA II sería más efectivo que amlodipino en disminuir la incidencia de infarto de miocardio, insuficiencia cardiaca congestiva y muerte cardiaca. El seguimiento fue de 1,450 pacientes que experimentan alguna variable principal. Se incluyeron más de 15,000 hipertensos mayores de 50 años con PA sistólica 160-210 y/o diastólica $95-115 \mathrm{mmHg}$ y riesgo cardiovascular alto o muy alto. De hecho, los pacientes incluidos son de población de riesgo muy alto: $33 \%$ son dislipémicos, $32 \%$ diabéticos, $24 \%$ fumadores y un alto número presentan enfermedad cardiovascular, $46 \%$ enfermedad coronaria, $20 \%$ con enfermedad cerebrovascular y $14 \%$ enfermedad vascular periférica. ${ }^{67}$

\section{CONSIDERACIONES}

Teniendo en cuenta el número de pacientes y el elevado porcentaje de factores de riesgo, los ARA II han demostrado buena eficacia y excelente tolerabilidad en el trata- miento de la HTA; un hecho trascendental es la disminución de la morbimortalidad cardiovascular superior a la de otros fármacos de probada eficacia antihipertensiva. Es de esperar que en un futuro próximo las indicaciones de los ARA II sean más amplias que las actuales, en la medida que estos fármacos sigan demostrando sus beneficios en la protección cardiovascular más allá del simple efecto del control de PA.

Recientemente se ha publicado un metaanálisis que recoge los estudios que comparan efectividad y tolerabilidad de IECA frente a ARA-II en pacientes con HTA esencial. Matchar et $\mathrm{al}^{7}$ seleccionaron 61 estudios clínicos que incluían al menos a 20 pacientes y tenían un tiempo de seguimiento mínimo de 12 semanas. El efecto antihipertensivo a largo plazo fue similar en ambos grupos farmacológicos. No se observaron diferencias en morbimortalidad cardiovascular, hipertrofia de ventrículo izquierdo, progresión a diabetes o enfermedad renal. Los efectos secundarios de los IECA fueron más pronunciados por la presencia de tos. La conclusión de los autores es que Ios IECA y Ios ARA-II tienen una efectividad antihipertensiva similar y los IECA, una peor tolerabilidad. Debe señalarse que el metaanálisis tiene numerosas limitaciones derivadas de la marcada heterogeneidad de los trabajos incluidos. En la mayoría, el número de pacientes analizados fue pequeño y los seguimientos superiores a un año, la excepción. Este estudio no responde adecuadamente a las preguntas básicas sobre estos dos grupos farmacológicos: ¿son igualmente efectivos IECA y ARA II? y ¿tienen efectos de protección vascular más allá de la reducción de las cifras de PA?

En relación con la efectividad antihipertensiva de la combinación IECA con ARA-II frente a la monoterapia, apenas se han publicado estudios. Aunque parecería que hay un pequeño efecto adicional, la comparación no se ha hecho con dosis altas de los monocomponentes. Además, la duración de los estudios ha sido muy breve (seis semanas) y el número de pacientes incluidos $<100$ en todos los casos. ${ }^{22}$ La publicación del estudio ONTARGET ha confirmado estas previsiones. En pacientes con riesgo vascular elevado, la mayoría de ellos hipertensos, el bloqueo dual con ramipril y telmisartán no ocasionó ningún beneficio añadido respecto a los monocomponentes. Por el contrario, hubo una mayor proporción de efectos adversos. ${ }^{23}$ Con estos datos, no se puede recomendar la combinación de un IECA con un ARA-II en HTA esencial.

Los IECA tienen grandes ventajas para el tratamiento de la HTA en diabéticos, así como para retrasar el desarrollo de glomerulopatía diabética y otras formas de gloméruloescrelosis. Los IECA se caracterizan por sus efectos clínicos benéficos demostrados por ensayos clínicos controlados aleatorizados de rigurosa calidad, como el estudio HOPE que demostró protección primaria de eventos cardiovasculares en pacientes diabéticos mayores de 55 años. Reducen la mortalidad por insuficiencia cardiaca sintomática, retrasan la progresión del daño renal en los diabéticos con microalbuminuria y re- 
ducen los eventos cardiovasculares de los pacientes en riesgo; ${ }^{68}$ sin embargo, los ARA II tienen mayor tolerabilidad y adherencia al tratamiento por tener menos efectos como la tos.

\section{BIBLIOGRAFÍA}

1. Organización Mundial de la Salud [OMS]. (2013). Global Health Observatory. Raised blood pressure. Situation and trends. de: http://www.who.int/gho/ncd/risk_factors/blood_ pressure_prevalence_text/en/index.html, recuperado el 01 enero de 2014.

2. Organización Mundial de la Salud [OMS]. (2012). ¿Es la hipertensión un problema frecuente?., de: http://new.paho.org/ col/index.php?option=com_content\&view=article\&id=190:his toria-del-dia-mundial-de-la-salud\&catid=794\&ltemid=500, Or ganización Mundial de la Salud [OMS].(2012). ¿Es la hipertensión un problema frecuente? Recuperado el 15 de febrero de 2014.

3. Velázquez Monroy $\mathrm{O}$ et al. Hipertensión arterial en México: Resultados de la Encuesta Nacional de Salud (ENSA) 2000. Arch Clin Mex. 2002; 72 (1): 71-84.

4. Jones DW, Appel LJ, Sheps SG, Roccella EJ, Lefant C. Measuring blood pressure accurately. New and Persistent Challenges. JAMA. 2003; 289 (8): 1027-1030.

5. Santos-Preciado JI, Villa-Barragán JP, García-Avilés MA et al. La transición epidemiológica de las y los adolescentes en México. Rev Sal Pub Mex. 2003; 45 (S1): S140-S152.

6. Omram AR. The epidemiology transition theory a preliminary. J Trop Pediatr. 1983; 29: 305-316.

7. Delgado RA, Fagundo MF, López LE et al. Transición epidemiológica. Rev Med Electrónica, Facultad de Ciencias Médicas, Matanzas, Cuba. 2003; 25 (1:1-6), tema 3 ene-feb. Recuperado 12 de enero de 2014.

8. Organización Mundial de la Salud [OMS]. (2002). Cuantificación de algunos riesgos importantes para la salud. En Informe Sobre la Salud en el Mundo 2002. Reducir los riesgos y promover una vida sana. http://www.who.int/whr/2002. Recuperado el 14 de febrero de 2014.

9. Martínez-Palomino G, Vallejo-Allende M, Huesca C, Álvarez de León $\mathrm{E}$, Paredes $\mathrm{G}$, Factores de riesgo cardiovascular en una muestra de mujeres jóvenes mexicanas, Rev Arch Cardiol Mex. 2006; 76 (4): 401-407.

10. Secretaría de Salud, México. Instituto Nacional de Salud Pública, Encuesta Nacional de Salud 2000, Tomo 2. La Salud de los Adultos, México, D.F., 2003:106-107.

11. Barquera S., Campos N. Ismael, Hernández B. L.,et al. Hypertension in Mexican adults: results from the National Health and Nutrition Survey 2006. Salud Publica Mex 2010 Vol 52: Suplemento I S63-S71.

12. Instituto Nacional de Salud Pública [INSP]. [2012). Encuesta Nacional de Salud y Nutrición (ENSANUT) 2012. http://ensanut.insp.mx/informes/ENSANUT2012ResultadosNacionales. pdf, Recuperado el 14 de febrero de 2014.

13. DGAE/DIE/SS, 2013. Comportamiento de la mortalidad en México 1995, 2000 y 2012.

14. Huerta RB, Factores de riesgo para la hipertensión arterial. Rev Arch de Cardiol de Mex. 2001; 71 (S1): S208-S210.

15. Ruilope LM, Barrios V. Pharmacologic treatment in hypertensive heart disease. In: Crawford MH, Di Marco JP eds. Cardiology. London: Mosby, 2001; 8.1-8.8.

16. Israili $\mathrm{ZH}$, Hall WD. Cough and angioneurotic edema associated with angiotensin-converting-enzyme inhibitor therapy: a review of the literature and pathophysiology. Am Intern Med. 1992; 117: 234-242.

17. Bloom BS. Continuation of initial antihypertensive medication after 1 year of therapy. Clin Ther. 1998; 20: 671-681.

18. Conlin PR, Gerth WC, Fox J, Roehm JB, Boccuzzi SJ. Fouryear persistence patterns among patients initiating theraphy with the angiotensina II receptor antagonist losartan versus other antihypertensive drug classes. Clin Ther. 2001; 23: 1999-2010.

19. Paster RZ, Snavely DB, Swett AR, Draper RA, Goldberg $\mathrm{Al}$, Soffer BA et al. Use of losartan in the treatment of hypertensive patients with a history of cough induced by angiotensin-converting-enzyme inhibitors. Clin Ther. 1998; 20: 978-989.

20. Black HR, Graff A, Shute D, Stoltz R, Ruff D, Levine J et al. Valsartan, a new angiotensin II antagonist of the treatment of essential hypertension. Efficacy, tolerability and safety compared to an angiotensin inhibitor. lisinopril. J Hum Hypertens. 1997; 11: 483-489.

21. Belcher G, Hubner R, George M, Elmfeldt D, lunde H: Candersartan cilexetil: Safety and tolerability in healthy volunteers and patients with hypertension. J Hum Hypertens. 1997; 11: 585-589.

22. Barrios V, Navarro A, Esteras A, Luque M, Romero J, Tamargo $\mathrm{J}$ et al. Antihypertensive efficacy and tolerability of lercarnidipine in daily clinical practice. The ELYPSE study. Blood Pressure. 2002; 11: 95-100.

23. Sever R. Candersartan cilexetil: a new, long-acting, effective angiotensin in type 1 receptor blocker. J Hum Hypertens. 1997; 11 (Suppl 2): 591-595.

24. Johnston CL. Angiotensin receptor antagonist: focus on losartan. Lancet. 1995; 346: 1403-1407.

25. Gillis JC, Markham A. Irbesartan: A review oí its pharmacodynamic and pharmacokinetic properties and therapeutic use in the management of hypertension. Drugs. 1997; 54: 885-890.

26. Markham A, Goa KL. Valsartan: a review of its pharmacology and therapeutic use in essential hypertension. Drugs. 1997; 54: 299-311.

27. Black HR, Graff A, Shute D, Stoiz R, Ruff D, Levine J et al. Valsartan, a new angiotensin II antagonist for the treatment of essential hypertension. Efficacy, tolerability and safety compared to an angiotensin- converting enzyme inhibitor, lisinopril. J Hum Hypertens. 1997; 11: 483-489.

28. Tikkanen I, Omvik P, Jensen HA, for the Scandinavian Study Group: Comparison of the angiotensin II antagonist losartan with the angiotensin converting enzyme inhibitor enalapril in patients with essential hypertension. J Hypertens. 1995; 13: 1343-1351.

29. Mimran A, Ruilope LM, Kerwin L, Nys M, Owens D, KasslerTaub $\mathrm{K}$ et al. A randomised, double blind comparison of the angiotensin II receptor antagonist irbesartan, with the full dose range of enalapril for the treatment of mild-to-moderate hypertension. J Hum Hypertens. 1998; 12: 203-208.

30. Kariberg BE, Lins LE, Hermansson K, for the TEES Study Group. Efficacy and Safety of Telmisartan a selectiva AT I receptor antagonist, compared with enalapril in ederly patients with Primary hypertension. J Hypertens. 1999; 17: 293-302.

31. Dahiof B, Keller S, Makris L, Goldberg AL, Sweet CS, Lim NY. Efficacy and tolerability of losartan potassium and atenolol in patients with mild or moderate essential hypertension. Am J Hypertens. 1995; 8: 578-583.

32. Corea L, Cardoni O, Fogari R, Innocenti P, Porcellati C, Providenza $\mathrm{M}$ et al. Valsartan, a new angiotensin II antagonist for the treatment of essential hypertension. A comparative study 
Enf Neurol (Mex) • 2014 • Vol. 13 • Núm. 3 • 139-146

of the efficacy and safety agonist amiodipine. Clin Pharm Ther. 1996; 60: 341-346.

33. Lombera F, Barrios V, Soria F, Placer L, Cruz JM, Tomás L et al. Guías de práctica clínica de la Sociedad Española de Cardiología en hipertensión arterial. Rev Esp Cardiol. 2000; 53: 66-90.

34. Guidelines Subcommittee. 1999 World Health Organization International Society of Hypertension guidelines for the management of hypertension. J Hypertens. 1999; 17: 151-183.

35. American Diabetes Association. Diabetic nephropathy. Diabetes Care. 2002; 25 (Suppl): S85-S89.

36. Kassier-Taub K, Little John T, Elliott W, Ruddy T, AdLer E. Comparative efficacy of two angiotensin II receptor antagonists, irbersartan and losartan, in mild to moderate hypertension. Am J Hypertens. 1998; 11: 445-453.

37. Andersson OK, Neldom S. The antihypertensive effect and tolerability of candersartan cilexetil, a new generation angiotensin II antagonist, in comparison with losartan. Blood Press. 1998; 7: 53-59.

38. Oparil S. Comparative antihypertensive efficacy of olmesartan: comparison with other angiotensin II receptor antagonists. J Hum Hypertens. 2002; 16 (suppl 2): 17-23.

39. Conlin P, Spence B, Ribeiro A, Saito I, Benedict C, Bunt M. Angiotensin II antagonist for hypertension: Are there differences in efficacy? Am J Hypertens 2000; 13: 418-426.

40. Hansson L, Lindhoim LH, Niskanen L, Lanke J, Hedner T, Niklason $\mathrm{A}$ et al. Effect angiotensin-converting-enzyme inhibition compared with conventional therapy on cardiovascular morbidity and mortality in hypertension: Captopril Prevention Project (CAPPP) randomised trial. Lancet. 1999; 353: 611616.

41. Brown MJ, Palmer CR, Castaigne A, de Leeuw PW et al. Mordidity and mortality in patients randomised to double-blind treatment with a long-acting calcium-channel blocker or diuretic in the internacional Nifedipine GITS study: Intervention as a goal in hypertension Treatment (INSIGHT). Lancet. 2000; 356: 366-372.

42. Hansson L, Hedner T, Lund-Johansen P, Kjeidsen S, Lindhoim LH, Syvertsen JO et al. Randomised trial of effects of calcium antagonists compared with diuretics and Beta-blockers on cardiocascular morbidity and morbility in hypertension: the Nordic Diltiazem (NORDIL) study. Lancet. 2000; 356: 359365.

43. Hansson L, Lindholm LH, Ekbom T, Dahiof B, Lanke J, Schersten B et al. Randomised trial of old and new antihypertensive drugs in elderly patients: cardiovascular mortality and morbidity the Swedish Trial in Old Patients with Hypertension-2-study. Lancet. 1999; 354: 1751-1756.

44. Lewis EJ, Hunsicker LG, Ciarke WR, Beri T, Pohi MA, Lewis JB et al. Collaborative Study Group. Renoprotective effect of the angiotensin-receptor antagonist irbesartan in patients with nephropathy due to type 2 diabetes. N Engl J Med. 2001; 345: 851-860.

45. Brenner BM, Cooper ME, de Zeeuw D, Keane WF, Mitch WE, Parving $\mathrm{HH}$ et al. Effects of losartan on renal and cardiovascular outcomes in patients with type 2 diabetes and nephropathy. N Eng J Med. 2001; 345: 861-869.

46. Dahlof B, Devereux RB, Kjeidsen SE et al. Cardiovascular morbidity and mortality in the losartan intervention for endpoint reduction in hypertension study (LIFE): a randomised trial against atenolol. Lancet. 2002; 359: 995-1003.

47. Dahlof B, Devereux RV, Julius $S$ et al. Characteristics of 9,194 patients with left ventricular hypertrophy: the LIFE study. Hypertension. 1998; 32: 989-997.
48. Dahlof $B$, Devereux RB, de Faire $U$ et al The losartan Intervention For Endpoint reduction (LIFE) in hypertension study: rationale, design, and methods. Am J Hypertens. 1997; 10: 705-713.

49. Dahlof B, Keller SE, Makris L, Goldberg Al, Sweet CS et al. Efficacy and tolerability of losartan potassium and atenolol in patients with mild to moderate essential hypertension. Am J Hypertens. 1995; 8: 578-583.

50. Dahlof B, Lindhoim LH, Hansson L, Schersten B, Ekbom T, Wester PO. Morbidity and mortality in the swedish trial in old patients with hypertension (STOP-Hypertension). Lancet. 1991; 338: 1281-1285.

51. Kjeldsen SE, Dahlof B, Devereux RB, Julius $S$ et al, for the LIFE Study Group. lowering of blood pressure and predictors of response in patients with left ventricular hypertrophy: the LIFE Study. Am J Hypertens. 2000; 13: 899-906.

52. Lindholm LH, Ibsen H, Dahlof B, Devereux RB et al. Cardiovascular morbidity and mortality in diabetics patients of the LIFE study: a randomised trial against atenolol. Lancet. 2002; 359: 1004-1010.

53. UK Prospective Diabetes Study Group. Efficacy of atenolol and captopril in reducing risk of macrovascular and microvascular complications in type 2 diabetes: UKPDS 39. BMJ. 1998; 317: 713-720.

54. Kjeldsen S, Dahlof B, Devereux R, Julius S, Aurup P et al, for the LIFE study. Effects of losartan on cardiovascular morbidity and mortality in patients with isolated systolic hypertension and left ventricular hypertrophy. A Losartan Intervention For Endpoint Reduction (LIFE) Substudy. JAMA. 2002; 288: 1491-1498.

55. Devereux RB, Palmieri B, Liu J et al. Progressive hypertrophy regression with sustained pressure reduction in hypertension: the Losartan Intervention For Endpoint Reduction Study. J Hypertens. 2002; 20: 1445-1450.

56. Dahiof B, Zanchetti A, Diez J et al. Effects of losartan and atenolol on left ventricular mass and neurhormonal profile in patients with essential hypertension and left ventricular hypertrophy. J Hypertens. 2002; 20: 1855-1864.

57. Wachtell K. Change in systolic left ventricular performance after 3 years of antihypertensive treatment: LIFE Study. Circulation. 2002; 106: 227-232.

58. Wachtell K. Change in diastolic left ventricular filling after one year of antihypertensive treatment: LIFE Study. Circulation. 2002; 105: 1071-1076.

59. Gerdts E. Correlates of pulse pressure reduction during antihypertensive treatment (losartan or atenolol) in hypertensive patients with electrocardiographic left ventricular hypertrophy (the LIFE Study). Am J Cardiol. 2002; 89: 399-402.

60. Gerdts E. Correlates of left atrial size in hypertensive patients with left ventricular hypertrophy: LIFE Study. Hypertension. 2002; 39: 739-743.

61. Wachtell K. Microalbuminuria in hypertensive patients with electrocardiographic left ventricular hypertrophy: the LIFE Study. J Hypertens. 2002; 20: 405-412.

62. Brunner HR, Gavras H. Angiotensin blockade for hypertension: a promise fulfilled. Lancet. 2002; 359: 990-992.

63. Barrios V, Campuzano R, Peña G et al. Estratificación del riesgo cardiovascular en hipertensión en Atención Primaria e impacto sobre el tratamiento antihipertensivo. Estudio DIORISC. Hipertensión. 2002; 19: 114-120.

64. Parving HH, Lehnert $\mathrm{H}$, Brochner-Mortensen J, Gomis R, Andersen S, Arner P. Irbesartan in patients with type 2 diabetes and microalbuminuria study group. The effect of irbesartan on 
the development of diabetic nephropathy in patients with type 2 diabetes. N Engl J Med. 2001; 345: 870-878.

65. Hansson L, Lithell $H$, Skoog $L$ et al. Study on cognition and prognosis in the elderly (SCOPE): baseline characteristics. Blood Press. 2000; 9: 146-151.

66. Mann J, Julius 5, for the VALUE Trial Group. The Valsartan Antihypertensive long-term. Use evaluation (VALUE) trial of cardiovascular events in hypertension. Rationale and design. Blood Press. 1998; 7: 176-183.

67. Kjeldsen SE, Julius S, Brunner $\mathrm{H}$ et al. A. Characteristics of 15314 hypertensive patients at high coronary risk. The VAL-
Enf Neurol (Mex) • 2014 • Vol. 13• Núm. 3•139-146

UE trial. The Valsartan Antihypertensive Long-term Use Evaluation. Blood Press. 2001; 10: 83-91.

68. Anastacio GD, Cornell KO, Menscer D. Drug interactions: Keeping it straight. Am Fam Physician. 2002; 66 (3): 461468.

Dirección para Correspondencia:

M. en C. Adela Alba-Leonel.

Antiguo Camino a Xochimilco y Viaducto Tlalpan, Col. San Lorenzo

Huipulco, 14370, Del. Tlalpan, México, D.F. Tel. 555556 2332, ext.

269, E-mail: adelaalbaleonel@yahoo.com.mx 